\title{
Effect of Adrenalectomy on Transport in the Rat Medullary Thick Ascending Limb
}

\author{
Jack Work and Rex L. Jamison \\ Division of Nephrology, University of Alabama at Birmingham, Birmingham, Alabama 35294; and Stanford University, Stanford, \\ California 94305
}

\begin{abstract}
Previous studies in adrenalectomized (Adx) rats suggest that aldosterone may regulate ion transport in the ascending portion of Henle's loop. In order to examine directly the effect of adrenalectomy on transport, medullary thick ascending limb (Mtal) segments were isolated from Adx, Adx replaced with aldosterone (Adx + Ald, $0.5 \mu \mathrm{g} \cdot 100 \mathrm{~g} \cdot$ body wt $\cdot \mathrm{d}$ ), and control Sprague-Dawley rats. Both net sodium and net chloride fluxes were significantly less in the Mtal segments from Adx rats compared with those in the control or Adx + Ald group. Physiologic levels of exogenous aldosterone increased net sodium chloride flux toward control values in the Adx + Ald group. Net potassium flux was not different among the three groups. We conclude that adrenalectomy impairs reabsorptive $\mathrm{NaCl}$ but not $\mathrm{K}$ transport in the Mtal, and that aldosterone restores this process. This reabsorptive defect may contribute to the urinary concentrating and diluting abnormality associated with adrenal insufficiency.
\end{abstract}

\section{Introduction}

It is generally agreed that a primary site of action of aldosterone is in the collecting duct. Recently, biochemical and functional evidence has accumulated that suggests the loop of Henle may also be an important target site for aldosterone. Horster and co-workers demonstrated that adrenalectomy caused a decrease in Na-K-ATPase in both thick ascending limb and collecting duct of rabbits (1). Aldosterone, when added in vitro to these nephron segments, restored $\mathrm{Na}-\mathrm{K}$ ATPase activity to normal within $1 \mathrm{~h}$ of incubation in both thick ascending limb and cortical collecting tubule. Marver and Lombard found that adrenalectomy reduced citrate synthase activity, a mitochondrial enzyme, in the medullary thick ascending limb. Moreover, acute administration of aldosterone returned activity to normal (2). Farman and Bonvalet have investigated specific aldosterone binding by the renal tubule of adrenalectomized rats (3). In these studies, the cortical collecting tubule was the more specific aldosterone target segment, but, significant aldosterone binding was demonstrated throughout the loop of Henle including the medullary thick ascending limb.

An effect of aldosterone on sodium chloride reabsorption

Address reprint requests to Dr. Work, Division of Nephrology, 643 Tinsley Harrison Tower, University Station, Birmingham, AL 35294.

Portions of this work have been published in abstract form, 1986. (Proc. Int. Union Physiol. Sci. 16:285a.)

Received for publication 27 January 1987 and in revised form 1 June 1987.

J. Clin. Invest.

(c) The American Society for Clinical Investigation, Inc.

0021-9738/87/10/1160/05 \$2.00

Volume 80, October 1987, 1160-1164 by the loop of Henle has been found in adrenalectomized rats using both free flow micropuncture and microperfusion techniques. In free-flow micropuncture studies by Hierholzer and co-workers, the distal tubule fluid-to-plasma (TF/P) sodium concentration was significantly higher in adrenalectomized rats than in intact rats (4). In a recent study by Stanton using in vivo microperfusion of the loop segment, adrenalectomy inhibited sodium reabsorption in the superficial loop of Henle; exogenous aldosterone replacement returned sodium transport to control levels (5).

These biochemical studies demonstrating specific aldosterone binding in the thick ascending limb of the loop of Henle and functional studies revealing a defect in sodium chloride transport in the thick ascending limb in adrenalectomized rats, suggest that aldosterone plays an important role in regulating sodium chloride transport in this segment of the nephron. The purpose of this study was to examine directly the effect of aldosterone on sodium chloride transport in the thick ascending limb.

\section{Methods}

Animals. The experiments were conducted using medullary thick ascending limb segments dissected from kidneys of barrier-maintained male Sprague-Dawley rats (Taconic Farms, Germantown, NY) weighing 60-125 g. The rats were housed in individual sterilized cages with filter covers and fed sterilized standard rat chow. Three groups of animals were studied: control rats with intact adrenal glands drinking tap water ad lib., adrenalectomized rats, and adrenalectomized rats administered exogenous aldosterone. Both groups of adrenalectomized rats were given $0.9 \% \mathrm{NaCl}$ to drink ad lib. instead of water. The adrenalectomized rats were obtained from the same supplier and kept for at least $\mathbf{4} \mathrm{d}$ before experimentation to allow endogenous hormone levels and steroid-dependent transporters to decrease to basal values. The adrenalectomized rats replaced with aldosterone received the hormone by means of an osmotic minipump (Alzet; Alza Corp., Palo Alto, CA) at a rate of $0.5 \mu \mathrm{g} / 100 \mathrm{~g}$ body wt per $\mathrm{d}$. This replacement dose was shown in previous studies to result in plasma levels of aldosterone similar to those measured in awake, unstressed rats fed the same diet $(6,7)$. This group of rats was kept for at least $4 \mathrm{~d}$ after implantation of the osmotic minipump (range, 4 to $6 \mathrm{~d}$ postimplantation of osmotic minipump) to allow aldosterone-dependent transporters to increase to steady state levels.

In vitro isolated perfused tubule studies. Rats were killed by decapitation immediately before dissection. Segments were isolated as previously described (8). Briefly, both kidneys were removed, decapsulated, and sectioned into 4 or 5 cross sections per kidney and immediately placed in a Petri dish containing bathing solution with $0.1 \%$ bovine albumin. Each section was stripped from the papillary tip to the cortex into smaller wedges and then transferred to a second dish under a dissection microscope. This minimized the number of tubule fragments that tend to obscure the dissection field. The corticomedullary junction and inner and outer stripe of outer medulla were identified and individual medullary thick ascending limbs were isolated by dissection.

The tubule segments were transferred to a thermo-regulated Lucite chamber containing albumin-free bathing solution (see below) initially 
at room temperature. After one end of the tubule was pulled into an outer holding pipette, an inner pipette containing the perfusate was advanced into the tubule lumen. The opposite end of the tubule was pulled into a glass micropipette for collecting the perfusate. The perfusion rate was maintained between 6 and $16 \mathrm{nl} / \mathrm{min}$ with an air reservoir pressure system. The experiments were carried out at $38^{\circ} \mathrm{C}$.

The bathing solution was a phosphate buffer solution containing the following (in mM): $10 \mathrm{Na}$ acetate, $5 \mathrm{KCl}, 5 \mathrm{NaH}_{2} \mathrm{PO}_{4} / \mathrm{Na}_{2} \mathrm{HPO}_{4}, 2$ $\mathrm{CaCl}_{2}, 1.2 \mathrm{MgCl}_{2}, 5.6 \mathrm{D}$-glucose and $6 \mathrm{~L}$-alanine. $\mathrm{NaCl}$ was added to bring the final osmolality to 310 mosmol. The bathing solution was adjusted to 7.4 and continuously bubbled with $100 \% \mathrm{O}_{2}$. The composition of the perfusate was identical to that of the bathing solution.

Transepithelial voltage measurements and analytical determinations. Perfusion rates were measured using dialyzed tritiated $\left[{ }^{3} \mathrm{H}\right]-$ methoxyinulin; the volume of collected fluid was measured with a constant bore pipette. Transepithelial voltage was measured between the perfusion pipette and the bathing solution using bridges containing $0.9 \% \mathrm{NaCl}$ and $4 \%$ agar connected via $3 \mathrm{M} \mathrm{KCl}$ tubing to calomel half cells. The half cells were connected to a differential amplifier, as described previously $(8,9)$.

The electron probe was used to determine the concentration of $\mathrm{Na}$, $\mathrm{K}$, and $\mathrm{Cl}$ in samples of the perfused and collected fluids. Samples obtained during perfusion were transferred and deposited under oil onto a Petri dish. The nanoliter sized drops were transferred with a 80 pl volumetric pipette and deposited under oil on a beryllium block. The oil was washed off with chloroform, leaving the deposited salts. The beryllium blocks were mailed in airtight containers. After their arrival at Stanford the samples were rehydrated by placing the beryllium block on a thermoelectric cooling plate (Cambion model 806-1001-01, Cambridge Thermionic Corp., Cambridge, MA) until ambient air began to condense on the surface of the block. The block was then placed on a piece of dry ice for 2-3 s to freeze the rehydrated droplets, and immediately transferred to a cold brass disk inside a small vacuum chamber to be freeze dried. The block was allowed to return to room temperature under vacuum for at least $2 \mathrm{~h}$.

The freeze-dried samples were analyzed at the Stanford University Center for Materials Research on a JEOL 733 electron microprobe, equipped with 5 wavelength dispersive spectrometers and automated by a DEC $11 / 23$ + computer. The counting conditions were as follows: accelerating voltage $15 \mathrm{kV}$; beam current $300 \mathrm{nA}$; beam diam 70-100 $\mu \mathrm{m}$; counting time $10 \mathrm{~s}$ for $\mathrm{Na}$ and $\mathrm{Cl}$ and $20 \mathrm{~s}$ for $\mathrm{K}$.

The procedure of sending volumetric, air-dried samples through the mail to be rehydrated, freeze-dried, and analyzed at their destination was tested by sending and analyzing four beryllium blocks containing 12-18 test samples each, on four separate occasions. The test samples were artificial solutions whose $\mathrm{Na}, \mathrm{Cl}$, and $\mathrm{K}$ concentrations had been measured by macro methods at Birmingham. The macro values were compared to those obtained by electron probe analysis. Table I summarizes the results of 55 consecutive comparisons of electron probe determinations in Stanford with macro determinations (flame photometer and chloridometer) in Birmingham.

The net flux of sodium, chloride, and potassium across the tubule epithelium was calculated as, $J_{\mathrm{i}}=\left(C_{0} V_{0}-C_{1} V_{1}\right) / L$, where $J_{\mathrm{i}}$ is the flux of the ith ion (pmol $\cdot \mathrm{min}^{-1} \cdot \mathrm{mm}^{-1}$ tubule length), $C_{0}$ is the concen-

Table I. Comparison of Electron Microprobe with Macro Determinations

\begin{tabular}{lllll}
\hline Element & $\mathrm{N}$ & Range of values & $\begin{array}{l}\text { Electron probe } \\
\text { to macro ratio }\end{array}$ & $\mathrm{SD}$ \\
\hline & & meq/liter & \\
Sodium & 55 & $12.0-191$ & 1.021 & 0.069 \\
Chloride & 55 & $15.6-227$ & 1.012 & 0.054 \\
Potassium & 55 & $1.2-24.2$ & 1.016 & 0.076 \\
\hline
\end{tabular}

Table II. Plasma Aldosterone

\begin{tabular}{lcl}
\hline Group & Number $^{*}$ & Plasma aldosterone $^{*}$ \\
\hline Adx & 17 & Undetectable \\
Adx + Aldo & 11 & $3.9 \pm 0.3$ \\
Intact & 9 & $3.3 \pm 1.6$
\end{tabular}

* Number of rats in each group.

‡ Plasma aldosterone (ng/dl, mean \pm SEM).

tration of the $\mathrm{i}^{\prime}$ th ion in the perfusion fluid $\left(\mathrm{pmol} \cdot \mathrm{nl}^{-1}\right), C_{1}$ is the $\mathrm{i}^{\prime}$ th concentration $\left(\mathrm{pmol} \cdot \mathrm{nl}^{-1}\right)$ in the collected fluid, $V_{0}$ is the perfusion rate $\left(\mathrm{nl} \cdot \min ^{-1}\right), V_{1}$ is the collection rate $\left(\mathrm{nl} \cdot \mathrm{min}^{-1}\right)$ and $L$ is length of the tubule $(\mathrm{mm})$. The mean value for each tubule sample was calculated from three to six measurements. Values for individual tubules were averaged to obtain the group means and to calculate the standard error of the mean.

To document the adequacy of aldosterone replacement, plasma aldosterone was measured in separate, identically treated groups of rats. Blood was collected from undisturbed, isolated animals by decapitation and plasma aldosterone was determined by radioimmunoassay (Coat-A-Count, Diagnostic Products, Los Angeles, CA).

To examine the effect of the short term exogenous aldosterone replacement on glomerular filteration rate, inulin clearance was measured in separate, identically treated groups of adrenalectomized rats and adrenalectomized rats administered exogenous aldosterone. Briefly, rats were anesthetized with Inactin (Byk Gulden, Konstanz, W. Germany), $100 \mathrm{mg} / \mathrm{kg}$ body wt i.p. and a tracheostomy tube was inserted to ensure adequate ventilation. Body temperature was maintained at $38^{\circ} \mathrm{C}$ throughout the clearance study. The right femoral artery and right external jugular vein were cannulated for the withdrawal of blood and the infusion of inulin, respectively. A bladder catheter was inserted to allow complete collection of urine. After a priming dose of tritiated inulin (New England Nuclear, Boston, MA) was given, a maintenance Ringer infusion containing sufficient isotope to provide $25 \mu \mathrm{Ci} / \mathrm{h}$ was started at a rate of $1 \mathrm{ml} / \mathrm{h}$. This was followed by an hour equilibration period prior to two 30 -min urine collections. Blood samples were obtained at the initiation and at the end of the urine collections. Inulin concentrations in urine and plasma were determined in a scintillation counter and urine volume was determined gravimetrically. Inulin clearance was calculated by the standard method.

Significance was estimated using a one way analysis of variance. The criterion for statistical significance was $P<0.05$. If there was a significant difference among means, the Scheffe test was used to evaluate the statistical significance between mean values (10).

\section{Results}

Plasma aldosterone levels for control (intact), adrenalectomized (Adx), ${ }^{1}$ and adrenalectomized rats replaced with exogenous aldosterone (Adx + Aldo) are given in Table II. Plasma aldosterone was undetectable in the Adx group. The replacement dose of aldosterone used in the Adx + Aldo group resulted in plasma aldosterone levels similar to those measured in the adrenal-intact group. There was no significant difference in glomerular filtration rate as determined by inulin clearance between the adrenalectomized and the adrenalectomized rats replaced with exogenous aldosterone (Table III).

Table IV summarizes the microperfusion data. The tubule

1. Abbreviations used in this paper: Adx, adrenalectomized; Aldo, aldosterone; GFR, glomerular filtration rate. 
Table III. Glomerular Filtration Rate

\begin{tabular}{lll}
\hline Group & Number* & GFR $^{\ddagger}$ \\
\hline Adx & 9 & $1.052 \pm 0.134$ \\
Adx + Aldo & 8 & $1.127 \pm 0.142$
\end{tabular}

* Number of rats in each group.

${ }^{\ddagger} \mathrm{ml} / \mathrm{min} / 100 \mathrm{~g}$ body wt (mean $\left.\pm \mathrm{SEM}\right)$.

segments for all groups averaged $0.49 \pm 0.04 \mathrm{~mm}$ in length (mean \pm SEM). There was no significant difference in collection rate among the three groups: $10.7 \pm 2.1,11.2 \pm 0.9$, and $13.5 \pm 1.2 \mathrm{nl} \cdot \mathrm{min}^{-1}$ for the Adx, Adx + Aldo, and intact groups, respectively. There was no significant fluid absorption by the tubules in any of the groups. Net chloride flux averaged $\sim 15 \%$ higher than net sodium flux. Both net fluxes were significantly less in the segments from adrenalectomized rats compared with those in the intact or Adx + Aldo group. Exogenous aldosterone treatment increased net sodium chloride flux toward control values (Table IV and Fig. 1). The transepithelial voltage was lumen-positive in all three groups but tended to be lower in the Adx group. However, only the Adx and the Adx + Aldo mean transepithelial voltages were significantly different (Table IV).

In contrast, net potassium fluxes varied widely and were not different between groups (Table IV).

\section{Discussion}

This study demonstrates directly that adrenalectomy impairs net sodium chloride absorption in the rat medullary thick as-

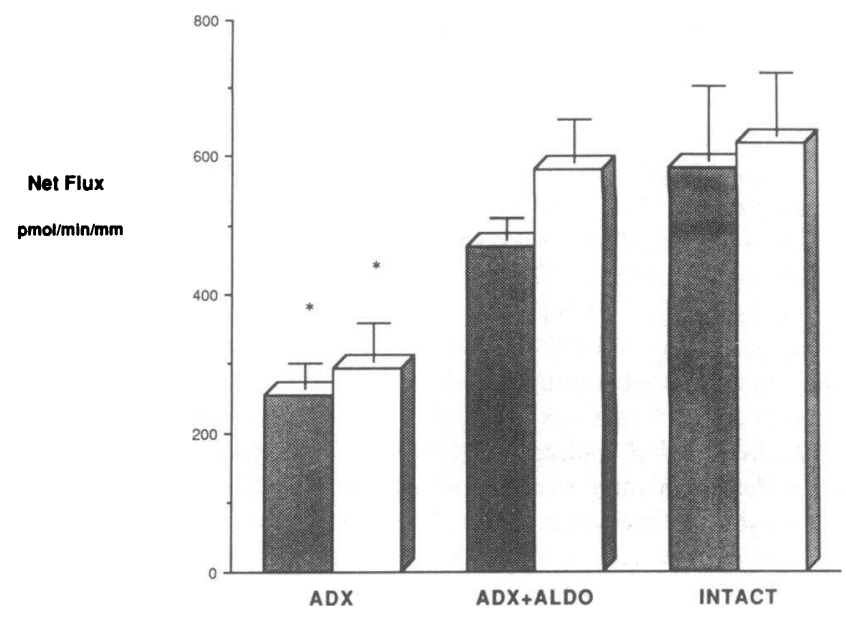

Figure 1. Effect of adrenalectomy on sodium and chloride net fluxes. ${ }^{*} P<0.05$ ADX versus ADX + ALDO and INTACT. Data represent mean \pm SE. $n$, Jnet Na; $\square, J$ net $\mathrm{Cl}$.

cending limb and physiological levels of aldosterone return net sodium chloride reabsorption toward normal. This reabsorptive defect in the medullary thick ascending limb may contribute to the impaired urinary concentrating and diluting ability (4) and to the salt wasting associated with adrenal insuffciency (11).

Previous observations suggest that aldosterone may regulate salt transport in the loop of Henle. To clarify why adrenal insufficiency impairs urinary dilution in the absence of antidiuretic hormone, Green and co-workers investigated the response to a water load in Brattleboro rats, a strain with hereditary hypothalamic diabetes insipidus (12). After adrenalec-

Table IV. Results of Microperfusion of the Medullary Thick Ascending Limb

\begin{tabular}{|c|c|c|c|c|c|c|c|}
\hline Group & Tubule & Length & Collection rate & Jnet $\mathrm{Na}$ & Jnet Cl & Jnet $\mathrm{K}$ & $V e$ \\
\hline & & $\mathrm{mm}$ & $n l / \min$ & $\mathrm{pmol} / \mathrm{min} / \mathrm{mm}$ & $\mathrm{pmol} / \mathrm{min} / \mathrm{mm}$ & $\mathrm{pmol} / \mathrm{min} / \mathrm{mm}$ & $m V$ \\
\hline \multirow[t]{5}{*}{ Adx } & 1 & 0.60 & 5.6 & 174 & 240 & 3.7 & 4.2 \\
\hline & 2 & 0.65 & 5.7 & 295 & 300 & 9.5 & 9.5 \\
\hline & 3 & 0.40 & 13.3 & 374 & 501 & 26.0 & 1.9 \\
\hline & 4 & 0.92 & 14.4 & 209 & 169 & 13.4 & 0.9 \\
\hline & 5 & 0.58 & 14.6 & 219 & 259 & 2.3 & 1.30 \\
\hline Mean \pm SEM & & $0.63 \pm 0.08$ & $10.7 \pm 2.1$ & $254 \pm 36$ & $294 \pm 56$ & $11.0 \pm 4.2$ & $2.1 \pm 0.7$ \\
\hline \multirow[t]{5}{*}{ Adx + Aldo } & 1 & 0.38 & 11.6 & 401 & 381 & 14.3 & 6.8 \\
\hline & 2 & 0.30 & 7.7 & 430 & 661 & -0.9 & 6.4 \\
\hline & 3 & 0.50 & 11.3 & 423 & 503 & 9.7 & 8.3 \\
\hline & 4 & 0.35 & 13.2 & 562 & 742 & 25.1 & 4.6 \\
\hline & 5 & 0.47 & 12.0 & 535 & 623 & 15.4 & 12.3 \\
\hline Mean \pm SEM & & $0.40 \pm 0.04$ & $11.2 \pm 0.9$ & $470 \pm 33$ & $582 \pm 63$ & $12.7 \pm 4.2$ & $7.7 \pm 1.3$ \\
\hline \multirow[t]{5}{*}{ Intact } & 1 & 0.44 & 9.9 & 316 & 447 & 0.4 & 8.2 \\
\hline & 2 & 0.61 & 15.2 & 867 & 729 & 24.7 & 2.7 \\
\hline & 3 & 0.48 & 11.9 & 487 & 397 & 5.7 & 4.6 \\
\hline & 4 & 0.45 & 16.4 & 817 & 895 & 15.8 & ND \\
\hline & 5 & 0.22 & 14.2 & 438 & 636 & 21.0 & 2.3 \\
\hline Mean \pm SEM & & $0.44 \pm 0.06$ & $13.5 \pm 1.2$ & $585 \pm 109$ & $621 \pm 91$ & $13.5 \pm 4.6$ & $4.6 \pm 1.3$ \\
\hline \multicolumn{8}{|l|}{ Statistical comparisons } \\
\hline Adx vs. intact & & NS & NS & $P<0.05$ & $P<0.05$ & NS & NS \\
\hline Adx vs. Adx + Aldo & & NS & NS & $P<0.05$ & $P<0.05$ & NS & $P<0.05$ \\
\hline Adx + Aldo vs. intact & & NS & NS & NS & NS & NS & NS \\
\hline
\end{tabular}


tomy, the rats were studied before and after mineralocorticoid or glucocorticoid replacement. Replacement of glucocorticoid alone fully restored the rats' capacity to excrete a water load but not their ability to maximally dilute the urine. Replacement of mineralocorticoid alone completely restored the diluting capacity, but not the capacity to excrete a water load; replacement of both steroids normalized both responses. These findings are consistent with the thesis that in the absence of aldosterone less sodium chloride is reabsorbed in the thick ascending limb so that less free water is generated.

The binding of aldosterone to its specific receptor in the tubule has been examined recently by Farmen and Bonvalet in the rat kidney (3). Although the cortical collecting tubule exhibited the highest specific nuclear labeling, significant specific aldosterone binding was demonstrated throughout the loop of Henle including the thin descending limb, thin ascending limb, and the medullary and cortical thick ascending limb segments. Cooke and Steenburg demonstrated that aldosterone administration significantly increased tissue sodium and urea concentrations in the outer medulla and papilla in adrenalectomized dogs (11). This is consistent with enhancement of sodium chloride reabsorption in the thick ascending limb of the loop of Henle.

Several studies have examined the effect of adrenalectomy on sodium transport in vivo by micropuncture or by microperfusion of superficial loops of Henle. Adrenalectomy results in increased tubule fluid-to-plasma $\mathrm{Na}$ ratios in the early distal tubule $(4,5,13)$ suggesting a defect in sodium reabsorption somewhere in the loop of Henle. Since the only segments that can lower the sodium concentration below that of the systemic plasma are the medullary and cortical thick ascending limbs, these findings suggest the impairment was in these segments. The results of studies examining the effect of exogenous aldosterone replacement in adrenalectomized rats, however, have been variable. Cortney failed to find an effect of exogenous aldosterone on sodium transport in the loop of Henle of the adrenalectomized rat (14). Murayama and co-workers could demonstrate normalization of sodium transport in the loop but only if cortisol was administered with aldosterone (13). Recently, Stanton has utilized in vivo microperfusion of the superficial loop segment to examine sodium transport by the loop of Henle (5). He found that physiological aldosterone replacement alone in the adrenalectomized rat restored sodium transport to normal. Possible explanations for the discordance in findings between the earlier studies $(13,14)$ and that by Stanton include alterations in glomerular filtration rate (GFR), tubule fluid flow rate, and solute delivery to the loop of Henle caused by adrenalectomy that were not controlled in the earlier studies. One advantage of the in vitro microperfusion technique employed in the present study is that these variables are all eliminated, which allows the effect of aldosterone on sodium chloride transport in the medullary thick ascending limb to become manifest.

Although the in vitro microperfusion technique eliminates several potential confounding variables, the augmentation in sodium chloride transport rate observed in the Adx + Aldo group could be an indirect effect of aldosterone secondary to an in vivo "conditioning effect" not seen in the Adx group. An increase in $\mathrm{NaCl}$ load to the medullary thick ascending limb secondary to an increase in GRF would represent such a conditioning effect, which could result in load-dependent increases in Na-K-ATPase activity in this segment. This in turn could cause the observed increase in the in vitro $\mathrm{NaCl}$ transport rate. However, GFR was not different with or without short term exogenous aldosterone replacement (Table III). Stanton and co-workers also found that short-term, physiologic replacement of aldosterone in adrenalectomized rats did not alter GFR (7). Thus, the increase in sodium chloride transport appears to be a direct effect of aldosterone on the medullary thick ascending limb.

Impairment of $\mathrm{NaCl}$ transport in the medullary thick ascending limb indicates why lack of aldosterone compromises both the ability to concentrate and dilute the urine. It also may play a role in the salt wasting that occurs in adrenocortical insufficiency. In vivo the loop of Henle is quantitatively the second most important tubule segment after the proximal convoluted tubule with regard to sodium reabsorption, accounting for $\sim 40 \%$ of reabsorption of the filtered load of sodium. Most of this presumably occurs in the medullary thick ascending limb. Based on comparative reabsorptive rates of $\mathrm{NaCl}$ by the medullary and cortical thick ascending limb segments in vitro, approximately twice as much $\mathrm{NaCl}$ is reabsorbed by the medullary thick ascending $\operatorname{limb}(15,16)$. Removal of aldosterone reduced $\mathrm{NaCl}$ reabsorption by twofold. One can estimate that instead of the usual delivery of $10 \%$ of the filtered load of $\mathrm{Na}$ to the beginning of the distal tubule, adrenalectomy would increase the delivery to 20 or $25 \%$. Even though adrenalectomy may result in a lower GFR (and therefore, filtered load of $\mathrm{Na}$ ), it is still very likely that the striking increase in $\mathrm{Na}$ delivery to the distal nephron, where $\mathrm{NaCl}$ transport capacity is limited, would contribute to salt wasting.

The generally accepted model of transport in the medullary thick ascending limb includes a secondarily active 1 potassium, 1 sodium, 2 chloride ions across the apical (luminal) membrane $(17,18)$. Thus, one might expect that impairment of sodium chloride transport in the medullary thick ascending limb by adrenalectomy, would also affect potassium transport. However, adrenalectomy did not alter net potassium transport. This incongruity may be explained by the recent observations of Hebert and collaborators that potassium is the principal conductive species in the apical membrane of the mouse medullary thick ascending limb (17). Their findings support the view that the majority of potassium efflux from cell to lumen through the apical potassium conductive pathway is recycled into cells by the sodium, potassium, 2-chloride cotransporter (16). Thus, adrenalectomy might be expected to reduce overall cycling of potassium across the apical membrane without changing net transepithelial potassium flux.

In summary, this study demonstrates that physiological levels of aldosterone modulate sodium chloride reabsorption in the medullary thick ascending limb. The reabsorptive defect in the medullary thick ascending limb caused by the lack of aldosterone may contribute to the urinary concentrating abnormality and the salt wasting associated with adrenal insufficiency.

\section{References}

1. Horster, M., H. Schmid, and U. Schmidt. 1980. Aldosterone in vitro restores nephron Na-K-ATPase of distal segments from adrenalectomized rabbits. Pfluegers Arch. Eur. J. Physiol. 384:203-206.

2. Marver, D., and W. E. Lombard. 1981. Localization of aldosterone-target sites in rabbit renal medulla. Kidney Int. 19:248A. 
3. Farman, N., and J. P. Bonvalet. 1983. Aldosterone binding in isolated tubules. III. Autoradiography along the rat nephron. Am. J. Physiol. 245:F606-F614.

4. Hierholzer, K., and S. Lange. 1974. The effects of adrenal steroids on renal function. In Kidney and Urinary Tract Physiology I. K. Thurau, editor. University Park, Baltimore. 6:273-333.

5. Stanton, B. A. 1986. Regulation by adrenal corticosteroids of sodium and potassium transport in loop of Henle and distal tubule of rat kidney. J. Clin. Invest. 78:1612-1620.

6. Martin, R. S., W. J. Jones, and J. P. Hayslett. 1983. Animal model to study the effect of adrenal hormones on epithelial function. Kidney Int. 24:386-391.

7. Stanton B., G. Giebisch, G. Klein-Robbenharr, J. Wade, and R. A. DeFronzo. 1985. Effects of adrenalectomy and chronic adrenal corticosteroid replacement on potassium transport in rat kidney. $J$. Clin. Invest. 75:1317-1326.

8. Work J., J. H. Galla, B. B. Booker, J. A. Schafer, and R. G. Luke. 1985. Effect of ADH on chloride reabsorption in the loop of Henle of the Brattleboro rat. Am. J. Physiol. 249:F698-703.

9. Work J., S. L. Troutman, and J. A. Schafer. 1982. Transport of potassium in the rabbit pars recta. Am. J. Physiol. 242:F226-F237.

10. Sokal, R. R., and F. J. Rohlf. 1981. Biometry. W. H. Freeman, San Francisco. 253-261.

11. Cooke, C. R., and R. W. Steenburg. 1973. Effects of aldosterone and cortisol on the renal concentrating mechanism. J. Lab. Clin. Med. 82:784-792.

12. Green, H. H., A. R. Harrington, and H. Valtin. 1970. On the role of antidiuretic hormone in the inhibition of acute water diuresis in adrenal insufficiency and the effects of gluco-and mineralocorticoids in reversing the inhibition. J. Clin. Invest. 49:1724-1736.

13. Murayama, Y., A. Suzuki, M. Tadokoro, M. Sakai, and F. Sakai. 1968. Microperfusion of Henles loop in the kidney of the adrenalectomized rat. Jpn. J. Pharmacol. 18:518-519.

14. Cortney, M. A. 1969. Renal tubular transfer of water and electrolytes in adrenalectomized rats. Am. J. Physiol. 216:580-598.

15. Burg, M. B., and J. E. Bourdeau. 1978. Function of the thick ascending limb of Henle's loop, in New Aspects of Renal Function. U. Vogel and K. Ullrich, editors. Excerpta Medica International Congress Series, No. 422. 91-102.

16. Knepper, M., and M. Burg. 1983. Organization of nephron function. Am. J. Physiol. 244:F579-F589.

17. Hebert, S. C., and T. E. Andreoli. 1984. Effects of antidiuretic hormone on cellular conductive pathways in mouse medullary thick ascending limbs of Henle: II. Determinants of ADH-mediated increases in transepithelial voltage and in net Cl-absorption. J. Membr. Biol. 80:221-223.

18. Greger, R. 1985. Ion transport mechanisms in thick ascending limb of Henle's loop of mammalian mephron. Physiol. Rev. 65:760797. 